\title{
Análise da composição físico-química da sujidade de plantas fotovoltaicas: estudo de caso para Fortaleza
}

\section{Analysis of the physical-chemical composition in photovoltaic plants: a case study for Fortaleza}

\author{
Análisis de la composición físico-química de la suciedad \\ de plantas fotovoltaicas: estudio de caso para Fortaleza
}

\section{Analyse de la composition physicochimique de la saleté des centrales photovoltaïques : étude de cas pour Fortaleza}

Marcelo Ferreira Freitas Filho

marceloferreira@alu.ufc.br Universidade Federal do Ceará (UFC)

Danielly Norberto Araujo danielly.araujo@ee.ufcg. edu.br Universidade Federal do Ceará (UFC)

Paulo Cesar Marques de Carvalho iD

carvalho@dee.ufc.br Universidade Federal do Ceará (UFC)

José Marcos Sasaki iD sasaki@física.ufc.br Universidade Federal do Ceará (UFC)

\section{Resumo}

Com o crescimento do setor fotovoltaico (FV), estudos que buscam aumentar a eficiência de módulos FV e analisar os fatores que impactam o seu desempenho vêm se intensificando. Sujidade é um dos parâmetros que interferem negativamente no desempenho dos módulos, cujo impacto depende não apenas da quantidade de sujeira acumulada, mas também da composição química e física da sujeira. Assim, o presente artigo tem como objetivo analisar a composição físico-química de amostras de sujidades coletadas de duas plantas FV localizadas na zona urbana de Fortaleza-CE. Na análise, as técnicas difração de raios-x (DRX), fluorescência de raios-x (FRX), microscopia eletrônica de varredura (MEV) e espetroscopia de energia dispersiva (EDS, Energy Dispersive Spectroscopy) são utilizadas para caracterização das sujidades. Por meio do DRX, as fases cristalinas identificadas são: $\mathrm{SiO}_{2}, \mathrm{Fe}_{2} \mathrm{O}_{3}, \mathrm{KAl}_{2}\left(\mathrm{AlSi}_{3} \mathrm{O}_{10}\right)(\mathrm{OH})_{2}, \mathrm{NaCl}, \mathrm{CaCO}_{3}$ e $_{6} \mathrm{H}_{8} \mathrm{O}_{6}$. Com o uso da FRX, os elementos encontrados com maior representatividade são: alumínio (8,87\% na planta 1 e $10,30 \%$ na planta 2), silício (36,67\% na planta 1 e $40,23 \%$ na planta 2$)$, ferro (19,55\% na planta 1 e $17,28 \%$ na planta 2$)$ e cálcio $(18,27 \%$ na planta 1 e $17,18 \%$ na planta 2$)$, o que é comum em áreas próximas a avenidas com grande fluxo de veículos. Utilizando o MEV, viu-se que o tamanho médio das partículas está na escala dos micrometros e, a partir da técnica EDS, que, além dos elementos encontrados pelo $\mathrm{FRX}$, também há a presença dos elementos carbono $(\mathrm{C})$, oxigênio $(\mathrm{O})$ e sódio $(\mathrm{Na})$ nas amostras.

Palavras-chave: Desempenho fotovoltaico. Sujidade. Composição físico-química. 
plant 2), which is common in areas close to avenues with great vehicle flow. Using SEM, it is seen that the average particle size is in the micrometer scale and from the EDS technique it is seen that in addition to the elements found by the FRX, there is also the presence of the elements carbon $(\mathrm{C})$, oxygen $(\mathrm{O})$ and sodium $(\mathrm{Na})$ in the samples.

Keywords: Photovoltaic performance. Soiling. Physico-chemical composition.

\section{Resumen}

Con el crecimiento del sector Fotovoltaico (FV), estudios que buscan aumentar la eficiencia de módulos FV y analizar los factores que impactan su rendimiento están intensificándose. Suciedad es uno de los parámetros que interfieren negativamente en el rendimiento de los módulos, cuyo impacto depende no solo de la cantidad de suciedad acumulada, pero también de la composición química y física de la suciedad. El presente trabajo tiene el objetivo de analizar la composición físico-química de muestras de suciedad recogidas de dos plantas FV ubicadas en la zona urbana de Fortaleza-CE. En el análisis, las técnicas Difracción de Rayos-X (DRX), Fluorescencia de Rayos-X (FRX), Microscopia Electrónica de Barrido (MEB) y Espectroscopia de Energía Dispersiva (EDS, Energy Dispersive Spectroscopy) son utilizadas para caracterización de las suciedades. Por medio del DRX, las fases cristalinas identificadas son: $\mathrm{SiO} 2, \mathrm{Fe} 2 \mathrm{O} 3, \mathrm{KAI}(\mathrm{AISi} 3 \mathrm{O} 10)(\mathrm{OH}) 2, \mathrm{NaCl}, \mathrm{CaCO} 3$ y $\mathrm{C} 6 \mathrm{H} 8 \mathrm{O} 6$. Con el uso de la FRX, los elementos encontrados con mayor representatividad son: aluminio $(8,87 \%$ en la planta 1 y $10,30 \%$ en la planta 2$)$, silicio (36,67\% en la planta 1 y $40,23 \%$ en la planta $2)$, hierro (19,55\% en la planta 1 y $17,28 \%$ en la planta 2$)$ y calcio (18,27\% en la planta 1 y $17,18 \%$ en la planta 2), lo que es común en áreas cercanas a avenidas con gran flujo de vehículos. Utilizando el MEB, se vio que el tamaño medio de las partículas está en la escala de los micrómetros y a partir de la técnica EDS se vio que además de los elementos encontrados por el FRX, también hay la presencia de los elementos carbono $(C)$, oxígeno (O) y sodio $(\mathrm{Na})$ en las muestras.

Palabras-clave: Rendimiento fotovoltaico. Suciedad. Composición físico-química.

\section{Résumé}

Avec la croissance du secteur photovoltaïque (PV), des études qui cherchent à améliorer l'efficacité des modules photovoltaïques bien comme ceux à analyser les facteurs qui ont un impact sur leurs performances ont connu une hausse. La saleté est l'un des paramètres qui interfère de manière négative dans les performances des modules. Son impact dépend de la quantité de saleté accumulée et de la composition chimique et physique de la saleté. Ainsi, cet article vise à analyser la composition physicochimique des échantillons de saleté prélevés sur deux centrales photovoltaïques situées dans la zone urbaine de Fortaleza, au l'état du Ceará, Brésil. Dans l'analyse, les techniques de diffraction des rayons $X$ (DRX), de fluorescence des rayons $X(X R F)$, de microscopie électronique à balayage (MEB) et de spectroscopie à dispersion d'énergie (SDE) sont utilisées pour caractériser les saletés. Grâce à DRX, les phases cristallines identifiées sont les suivantes : $\mathrm{SiO} 2, \mathrm{Fe} 2 \mathrm{O} 3, \mathrm{KAl}$ (AISi3O10) $(\mathrm{OH}) 2, \mathrm{NaCl}, \mathrm{CaCO} 3$ et $\mathrm{C} 6 \mathrm{H} 8 \mathrm{O} 6$. Avec l'utilisation de XRF, les éléments trouvés avec la plus grande représentativité sont : aluminium (8.87\% dans la centrale 1 et $10.30 \%$ dans la centrale 2 ), silicium ( $36.67 \%$ dans la centrale 1 et $40.23 \%$ dans la centrale 2$)$, fer $(19.55 \%$ dans la centrale 1 et $17.28 \%$ dans la centrale 2$)$ et calcium ( $18.27 \%$ dans la centrale 1 et $17.18 \%$ dans la centrale 2), ce qui est commun dans des secteurs proches à des avenues avec grand flux de véhicules. Quand on a utilisé le MEB, on a vu que la dimension moyenne des particules se trouve dans l'échelle des micromètres. La technique SDE a montré que, au-delà des éléments trouvés par la FRX, il y a aussi la présence des éléments carbone $(\mathrm{C})$, oxygène $(\mathrm{O})$ et sodium $(\mathrm{Na})$ dans les échantillons.

Mots-clés: Performance photovoltaïque. Saleté. Composition physicochimique. 


\section{Introdução}

É notável o crescimento da participação da energia solar por meio da tecnologia FV na matriz elétrica mundial e nacional. Segundo a IEA (International Energy Agency), a capacidade instalada FV mundial ultrapassou a marca de 500 GW em 2018 (IEA, 2019), enquanto no Brasil atingiu uma potência instalada de 1.798 MW e foi responsável por $3.461 \mathrm{GWh}$ de energia elétrica produzidos no mesmo ano. Além disso, no Brasil, entre os anos de 2017 e 2018, houve um aumento de 92,2\% da potência instalada FV e de $316,1 \%$ da produção de energia elétrica por meio de módulos FV (Empresa de Pesquisas Energéticas (EPE), 2019).

Consequentemente, devido ao aumento do uso da tecnologia FV para geração de energia elétrica, estudos relacionados aos parâmetros que afetam o desempenho de módulos têm aumentado substancialmente. $\mathrm{O}$ propósito desses estudos é investigar as causas, os impactos e as formas de mitigação ou minimização dos efeitos causados pelos parâmetros influenciadores no desempenho FV. Os principais fatores que influenciam o desempenho dos módulos são: intensidade de irradiação solar, temperatura do ar, sujidade, umidade relativa e vento (KAZEM; CHAICHAN, 2016).

Sujidade é o terceiro parâmetro ambiental que mais afeta o desempenho de plantas FV, sendo apenas menos relevante do que a irradiação solar e a temperatura, e é devido ao acúmulo de partículas de poeira ou de outros contaminantes na superfície FV (MICHELE et al. 2019). As partículas de sujidade acumuladas na superfície do módulo formam uma camada de sujeira que reduz, ou até mesmo bloqueia, a incidência de irradiação solar. O grau da influência da sujidade no desempenho dos módulos FV pode variar de local para local, podendo causar, nos piores cenários, uma redução na potência de saída FV de até 78\% (COSTA et al., 2018). Na Figura 1 são mostrados os módulos FV em condições de sujidade.

Figura 1 - Sujidade em módulos FV.

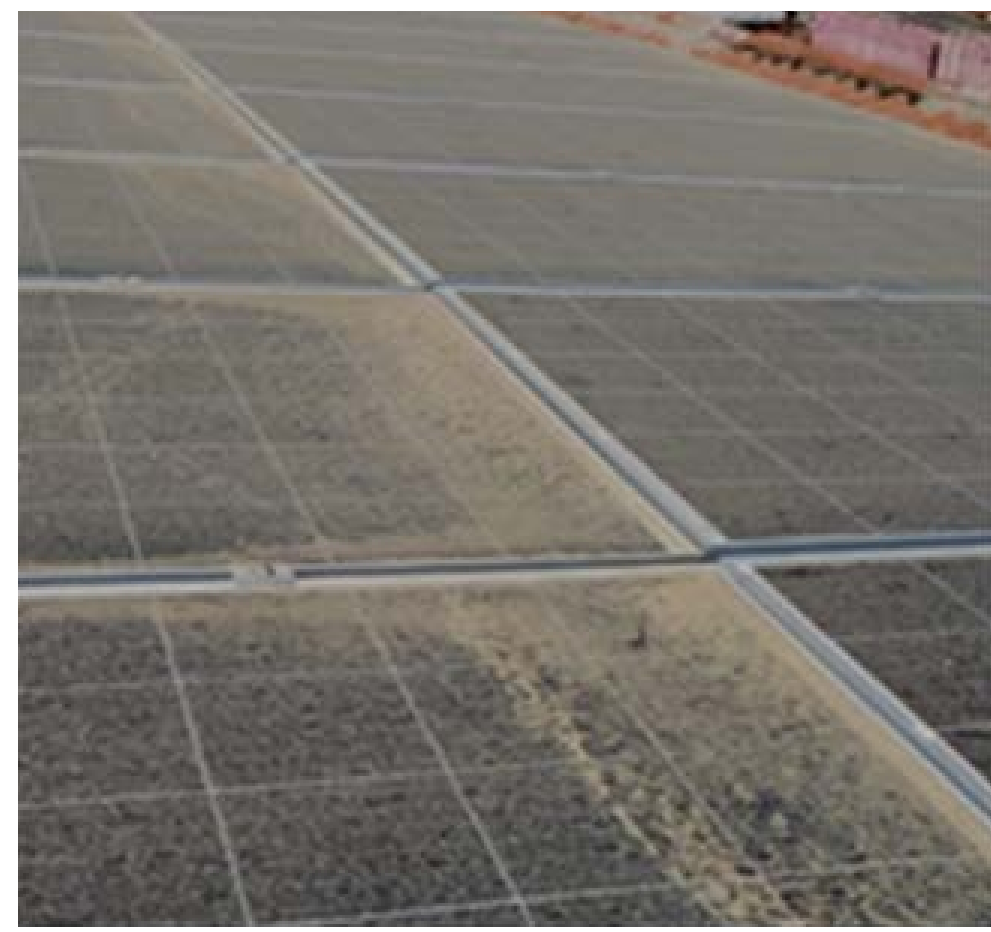

Fonte: Elaborada pelos autores, 2020. 
A redução no desempenho das células FV devido à sujidade depende não apenas da quantidade de ssujidade acumulada, mas também da sua composição físico-química (SAIDAN et al., 2016). A composição da sujidade depositada sobre os módulos FV varia em todo o mundo, sendo esperado que a sua composição esteja fortemente ligada à composição da sujidade da região/solo/terreno local (LAWRENCE; NEFF, 2009).

Além disso, as perdas de transmissão de luz causadas pela sujidade nas superfícies dos módulos são fortemente influenciadas pelo tamanho e distribuição das partículas nos módulos FV, apresentando perdas significativamente maiores por acúmulo de sujeira para partículas de tamanhos menores (SARVER et al., 2013). Isso pode ser explicado pelo fato de que partículas menores são distribuídas mais uniformemente do que partículas maiores, o que leva a uma maior dispersão de luz.

Pesquisas realizadas a partir da deposição de sujidade natural permitem prever perdas de sujidade e ainda identificar os níveis de sujidade em diferentes regiões. Além disso, também são essenciais para o desenvolvimento de tecnologias de mitigação de sujidade, pois a composição química e a distribuição do tamanho das partículas determinam como a sujidade interage com a superfície FV e, portanto, afeta a aplicabilidade e a eficácia das técnicas de mitigação (JAVED et al., 2017).

Araújo et al. (2019) analisaram 23 trabalhos sobre o impacto da sujidade em módulos FV, sendo 9 nacionais e 13 internacionais, e identificaram que: $65,22 \%$ das pesquisas não realizaram o estudo da composição química da sujidade em módulos FV e 73,91\% não investigaram o tamanho das partículas. Dos trabalhos analisados, os autores perceberam que $91,30 \%$ utilizaram a forma natural de deposição de sujidade no estudo.

O presente trabalho tem como objetivo avaliar a composição físico-química da sujidade depositada em módulos FV instalados na zona urbana de Fortaleza, Ceará. Assim, por meio de amostras de sujidades coletadas em módulos FV caracterizados pela deposição natural de sujidade, a composição química e o tamanho das partículas das sujidades serão identificados e analisados.

\section{Estado da arte}

Na presente seção, são descritos os resultados de artigos científicos que abordam o estudo da composição da sujidade em módulos FV, retratando, entre outros aspectos, as características de instalação das plantas FV estudadas e o modo como a avaliação da sujidade foi realizada. O estado da arte é realizado a fim de poder comparar os resultados encontrados no presente artigo com os resultados de outros trabalhos.

Costa et al. (2018) apresentam a caracterização físico-química de diferentes amostras de sujidades provenientes de módulos FV instalados, há mais de 10 anos, nas cidades de Diamantina, Montes Claros e Sete Lagoas, em Minas Gerais, Brasil. Para a coleta da sujidade dos módulos, uma pá plástica é utilizada para raspagem das amostras de sujeira. Na caracterização, as técnicas EDS e MEV são aplicadas. Em relação ao EDS, o ensaio indica que na cidade de Diamantina há maiores concentrações de caulinita, quartzo e hematita nas amostras de sujidade, enquanto em Montes Claros são predominantes calcita e gipsita e, em Sete Lagoas, é dióxido de titânio. Quanto ao MEX, o teste mostra que, em Diamantina, predominam partículas de tamanho de $10 \mu \mathrm{m}$ e elementos como carbono, oxigênio, ferro, alumínio, silício, enxofre e cálcio; em Montes Claros, partículas esféricas de hematita, com dimensões próximas a $4 \mu \mathrm{m}$, são predominantes; em Sete Lagoas, predominam partículas com maior brilho constituídas por hematita.

Menoufi et al., (2017) apresentam um estudo sobre módulos posicionados no telhado do Laboratório de Energias Renováveis da Universidade Beni-Suef, em Beni-Suef, Egito. No experimento, dois módulos FV multicristalinos, de $10 \mathrm{Wp}$ cada, são expostos ao ambiente externo por três meses. Os módulos FV são colocados em um ângulo de inclinação de $0^{\circ}$, sendo um limpo diariamente e o outro deixado para acúmulo contínuo de poeira. A densidade de sujeira encontrada no módulo devido ao tempo de exposição é de, aproximadamente, $0,2545 \mathrm{~g} / \mathrm{cm}^{2}$, com o tamanho da partícula variando entre 3,5 e $30 \mu \mathrm{m}$.

Conceição et al. (2018) avaliam o impacto do transporte de poeira do deserto do Saara em plantas FV que estão localizadas em dois locais da zona rural de Portugal, em Alter do Chão e Évora. Para análise, 
vidros de alta transmitância são instalados nos locais em que as plantas FV estão localizadas. O maior acúmulo medido é em fevereiro de 2017, sendo de $402 \mathrm{mg} / \mathrm{m}^{2}$, em Alter do Chão, e de $1067 \mathrm{mg} / \mathrm{m}^{2}$, em Évora. Analisando a sujidade, é visto que, em Alter do Chão, a sujeira se apresenta de forma aglomerada e é composta, principalmente, por aluminos silicatos e halita. Em Évora, as partículas de sujeira se apresentam espalhadas e os principais componentes são calcita e quartzo. Analisando o impacto da sujidade no desempenho dos módulos FV, a maior queda de potência de saída e da corrente de curto circuito acontece em Évora: $8 \%$, contra 3\% em Alter do Chão.

Gholami et al. (2018) realizam um experimento em uma planta FV de 14,5 kWp, composta por 57 módulos monocristalinos, divididos em 3 strings e instalada na Universidade Shahid Beheshti, em Teerã, Irã. Para medição do acúmulo de sujidade na superfície dos módulos FV, há a medição semanal desse parâmetro, sendo observado que, após 70 dias sem chover, embora o acúmulo de sujeira tenha aumentado, a deposição de sujeira é mais rápida nas primeiras semanas do experimento. Para um acúmulo de sujeira na superfície do módulo de 6,0986 $\mathrm{g} / \mathrm{m}^{2}$, há uma redução na potência de saída de $21,47 \%$. A corrente de curto-circuito diminui significativamente à medida que a densidade de sujeira aumenta. Apesar de a tensão de circuito aberto também diminuir, não é tão perceptível quanto o efeito da sujeira na corrente de curto-circuito. Na composição da sujeira, os principais compostos encontrados foram: dióxido de silício, óxido de cálcio, óxido de alumínio e óxido de ferro.

Romanholo et al. (2018) analisam as características físico-químicas da sujidade depositada sobre os módulos de uma planta FV na Universidade Federal de Goiás, Brasil. A planta contém 145 módulos do tipo policristalino e possui uma potência instalada de $34 \mathrm{kWp}$. A sujeira dos módulos foi coletada via raspagem com bastonetes de algodão. Foram coletadas cinco amostras de diferentes módulos (amostra 1: regiões esbranquiçadas nos módulos; amostra 2: manchas escuras nos módulos; amostra 3: sujidade uniformemente distribuída sobre os módulos; amostra 4: sujidade volumosa no módulo da central meteorológica; amostra 5: material sedimentado sobre o módulo da central meteorológica) e a sujidade encontrada apresentou componentes e tamanhos diferentes em cada uma dessas amostras. Na amostra 1, há alta concentração de silício, alumínio, magnésio. Nas amostras 2 e 3, há alta concentração de carbono e cálcio. A amostra 4 apresenta óxido de enxofre, e a amostra 5 apresenta carbono, oxigênio, cálcio e nitrogênio. Em relação ao tamanho das partículas, as amostras 1 e 4 apresentam sujidade aglomerada, o que dificultou a estimativa do tamanho, já as amostras 2 e 3 possuem partículas menores do que $10 \mu \mathrm{m}$, e a amostra 5 possui partículas bem menores, na ordem de $100 \mu \mathrm{m}$.

Ferrada et al. (2019) caracterizam as propriedades químicas e físicas da sujidade depositada em módulos FV de 4 locais no deserto de Atacama, Chile. Os locais são distantes $100 \mathrm{~km}$ um do outro e são denominados como L1 (localização mais ao norte), L2 (zona industrial), L3 (costa) e L4 (localização mais ao sul), sendo que L1, L2 e L4 possuem clima árido e L3, clima hiperárido. A caracterização da sujeira é realizada a partir de amostras do solo e da superfície dos módulos. Em L1 e L2, as partículas de tamanho entre 1 e 10 $\mu \mathrm{m}$ são mais comumente encontradas no solo do que nos módulos, sendo em L2, por exemplo, $75 \%$ e $56 \%$ a concentração dessas partículas no solo e no módulo, respectivamente; em L3 e L4, o comportamento é diferente, a concentração de partículas de tamanho entre 1 e $10 \mu \mathrm{m}$ é maior no módulo, sendo em L4 de $76 \%$ e $60 \%$ a concentração dessas partículas no solo e no módulo, respectivamente. Na composição química, os principais elementos comuns a todas as localizações são oxigênio e silício, mas também há cálcio, sódio e cloro. Os minerais comuns são albita, anortita, quartzo e ortoclásio, mas também há outros que são específicos do local, como mostrado na Tabela 1. 
Tabela 1 - Minerais presentes nas amostras de sujeira coletadas da superfície do módulo (M) e do solo (S) nas localizações L1 - L4.

\begin{tabular}{l|c|c|c|c|c|c|c|c}
\hline Mineral & L1-M & L1-S & L2-M & L2-S & L3-M & L3-S & L4-M & L4-S \\
\hline Albita & $\mathrm{X}$ & $\mathrm{X}$ & $\mathrm{X}$ & $\mathrm{X}$ & $\mathrm{X}$ & $\mathrm{X}$ & $\mathrm{X}$ & $\mathrm{X}$ \\
\hline Anortita & $\mathrm{X}$ & $\mathrm{X}$ & $\mathrm{X}$ & $\mathrm{X}$ & $\mathrm{X}$ & $\mathrm{X}$ & $\mathrm{X}$ & $\mathrm{X}$ \\
\hline Calcita & & & & & $\mathrm{X}$ & $\mathrm{X}$ & & \\
\hline Cristobalita & $\mathrm{X}$ & $\mathrm{X}$ & & & $\mathrm{X}$ & $\mathrm{X}$ & & \\
\hline Gipsita & & & $\mathrm{X}$ & $\mathrm{X}$ & & & & \\
\hline Halita & & & $\mathrm{X}$ & $\mathrm{X}$ & $\mathrm{X}$ & $\mathrm{X}$ & & \\
\hline Quartzo & $\mathrm{X}$ & $\mathrm{X}$ & $\mathrm{X}$ & $\mathrm{X}$ & $\mathrm{X}$ & $\mathrm{X}$ & $\mathrm{X}$ & $\mathrm{X}$ \\
\hline Muscovita & $\mathrm{X}$ & $\mathrm{X}$ & $\mathrm{X}$ & $\mathrm{X}$ & & & & \\
\hline Ortoclásio & $\mathrm{X}$ & $\mathrm{X}$ & $\mathrm{X}$ & $\mathrm{X}$ & $\mathrm{X}$ & $\mathrm{X}$ & $\mathrm{X}$ & $\mathrm{X}$ \\
\hline Focio
\end{tabular}

Fonte: Ferrada et al,. 2019.

Hachicha, Al-Sawafta e Said (2019) investigam a sujidade e seus efeitos em módulos FV instalados em Sharjah, nos Emirados Árabes Unidos, dividindo o estudo em três partes. A primeira parte é realizada em laboratório, utilizando uma lâmpada solar para simular o sol, um módulo policristalino de 5 W e amostras de sujeira coletadas do ambiente externo. As amostras de sujeira variam entre 0,0063 g e 0,34 g, e são homogeneamente espalhadas na superfície do módulo. As curvas I-V e P-V do módulo FV são traçadas antes e depois das amostras de sujeira ser espalhadas. Uma relação linear é obtida entre a potência FV normalizada e o acúmulo de sujeira na superfície do módulo, com uma queda de $1,7 \%$ por $\mathrm{g} / \mathrm{m}^{2}$. Além disso, ao aumentar a densidade de sujeira de zero para $8 \mathrm{~g} / \mathrm{m}^{2}$, a Icc é mais afetada do que Voc.

Na segunda parte, experimentos em condições externas são conduzidos, entre 11/01/2018 e 5/07/2018, em três módulos policristalinos de $5 \mathrm{~W}$ com diferentes ângulos de inclinação $\left(0^{\circ}, 25^{\circ} \mathrm{e} 45^{\circ}\right)$. Para comparação de eficiência, um módulo limpo de mesma inclinação foi colocado ao lado do módulo sujo. Após duas semanas de exposição ao ar livre, a queda na eficiência aumentou em 37,63\%, 14,11\% e 10,95\% para os módulos inclinados em $0^{\circ}, 25^{\circ}$ e $45^{\circ}$, respectivamente. Após cinco meses de exposição, a perda de potência do módulo devido à sujidade aumentou de $0,29 \%$ para $12,7 \%$, e a densidade de sujeira de $0 \mathrm{~g} / \mathrm{m}^{2}$ para $5,44 \mathrm{~g} / \mathrm{m}^{2}$. Na última parte do experimento, a caracterização da sujidade é realizada, sendo observadas partículas de sujeira com tamanhos entre 1,61 e 38,40 $\mu \mathrm{m}$ de diâmetro, sendo $95 \%$ das partículas com tamanho menor do que 25 $\mu \mathrm{m}$ de diâmetro. Os principais elementos encontrados nas partículas de sujeira são oxigênio, carbono, cálcio e silício, e os principais minerais são dióxido de silício, óxido de cálcio e óxido de ferro.

Tanesab et al. (2019) investigam os efeitos da sujidade em módulos FV de diferentes tecnologias: silício amorfo, mono e policristalino, e potências de 119,35 Wp, 76,04 Wp e 29,21 Wp, respectivamente, instalados em Babuin, na Indonésia, e em Perth, na Austrália. As superfícies dos módulos são sujas artificialmente, a partir da pulverização de uma mistura de água e de sujeira do solo do local. Para análise do desempenho dos módulos, suas curvas I-V são traçadas antes e depois do processo de sujeira. Caracterizando a sujidade dos módulos FV, os principais elementos da sujeira em Perth são: oxigênio, silício e cálcio; e, em Babuin, são: cálcio, oxigênio e ferro. Os principais compostos minerais nos dois locais são: quartzito e óxido de cálcio. Em relação ao tamanho das partículas de sujeira, em Babuin há maior concentração de partículas do que em Perth, que possui menor concentração de argila (sujeira com diâmetro $<4 \mu \mathrm{m}$ ), como pode ser visto na Figura 2. Além disso, o desempenho de cada tecnologia FV nos dois locais, para uma quantidade equivalente de sujidade, é semelhante.

\section{Metodologia}

\subsection{Localização das plantas FV}

As duas plantas FV estudadas estão localizadas no Laboratório de Energias Alternativas (LEA) da Universidade Federal do Ceará (UFC), ambas com um ângulo de inclinação em relação à superfície de $10^{\circ}$. 
As plantas estão situadas próximas à avenida Mister Hull, que possui grande fluxo de veículos, o que agrava o acúmulo de sujidade. A planta FV 1 foi instalada em 2016 e possui 6 módulos FV de $250 \mathrm{Wp}$, totalizando uma potência instalada de 1,5 kWp. Já a planta FV 2 possui 12 módulos de FV de $325 \mathrm{Wp}$, distribuídos em duas strings com 6 módulos, totalizando uma potência instalada de 3,90 kWp. Na Figura 2 são apresentadas as plantas FV instaladas no LEA-UFC (FREITAS FILHO, 2019).

Figura 2 - Visão aérea das plantas FV instaladas no LEA - UFC.

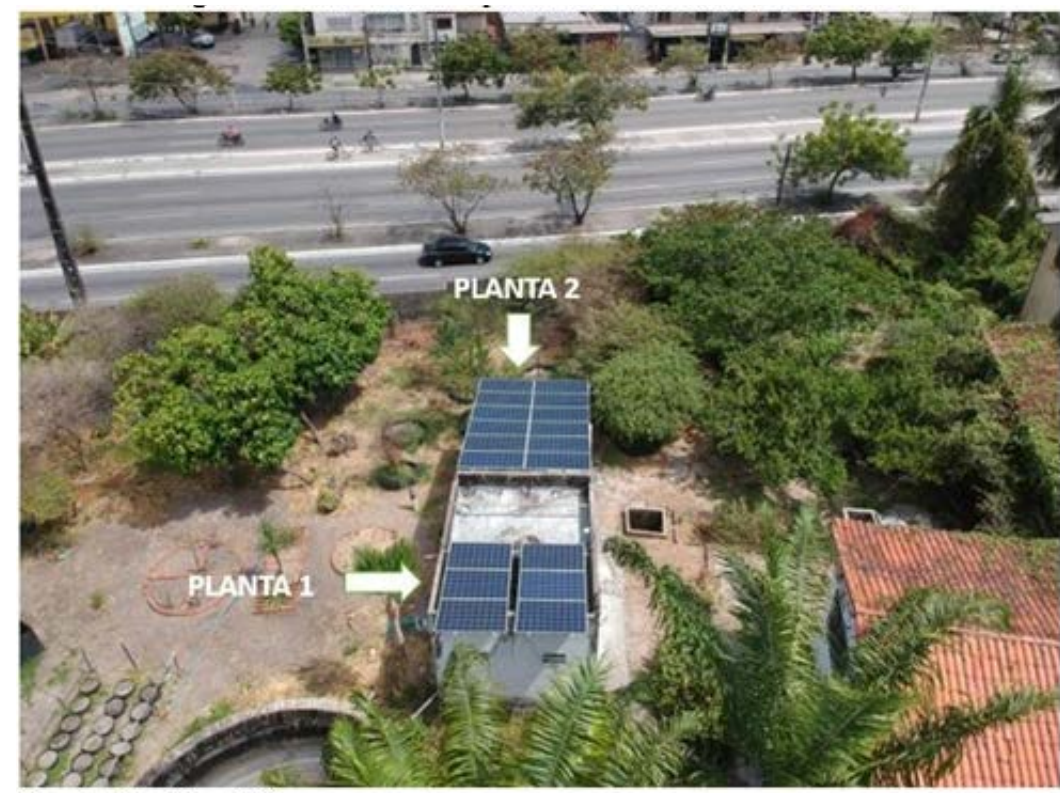

Fonte: Freitas Filho, 2019.

A coleta das amostras de sujidade das duas plantas foi realizada no dia 30/09/2019. A última chuva registrada anterior ao dia mencionado foi no dia 22/09/2019, de 0,20 $\mathrm{mm}$. A sujidade se apresentou de forma concentrada nas bordas inferiores dos módulos das plantas FV, indicando que a chuva registrada no dia 22/09/2019 não possuiu efeito de limpeza. Além disso, dejetos de pássaros foram encontrados. Para a coleta da sujidade, foram utilizados um pincel, uma espátula de plástico e um recipiente, mostrados na Figura 3 , a fim de manter a amostra sem contaminação. Há de se destacar o uso de luvas (FREITAS FILHO, 2019).

Figura 3 - Processo de coleta da sujidade

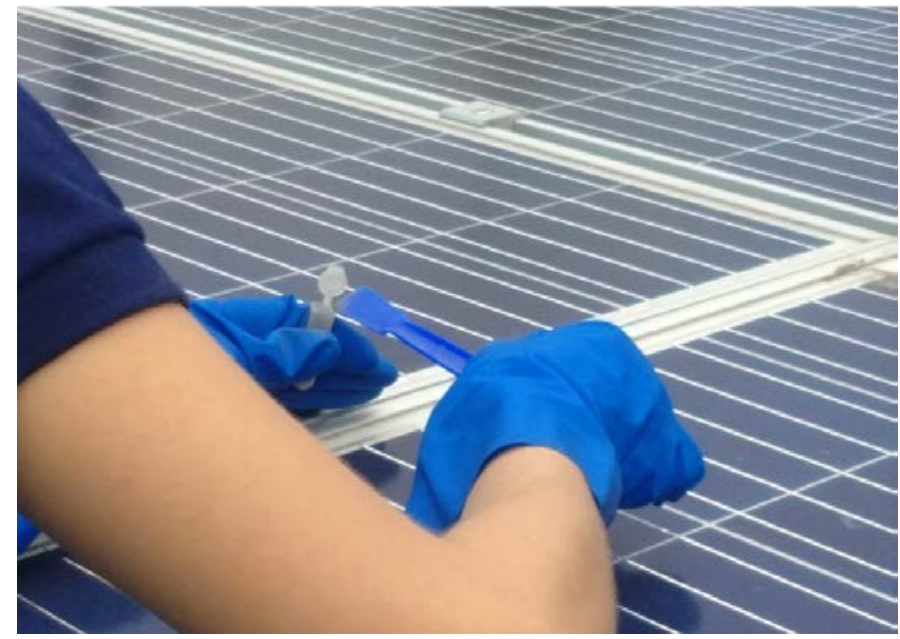

Fonte: Freitas Filho, 2019. 


\subsection{Ensaios realizados}

Para caracterização da sujidade, o presente estudo teve colaboração do Laboratório de Raios X (LRX) e da Central Analítica, ambos localizados na UFC. As técnicas FRX e DRX foram aplicadas no LRX, enquanto as técnicas MEV e EDS foram aplicadas na Central Analítica.

\subsubsection{FRX}

Os ensaios de FRX foram realizados numa ZSXMini II - Rigakuß, Figura 4, que identifica a presença de elementos químicos da tabela periódica desde o flúor até o urânio, o que demonstra uma restrição espectral na detecção da energia emitida por determinados elementos químicos. Essa técnica permite identificar os elementos presentes em uma amostra e determinar a concentração de cada elemento de maneira semiquantitativa. Uma fonte de radiação de elevada energia (raios X) provoca a excitação dos átomos da substância que se pretende analisar. O princípio de funcionamento do equipamento se baseia no salto que o elétron realiza de uma camada mais externa para uma mais interna de maior energia. (CALLISTER; RETHWISCH, 2012).

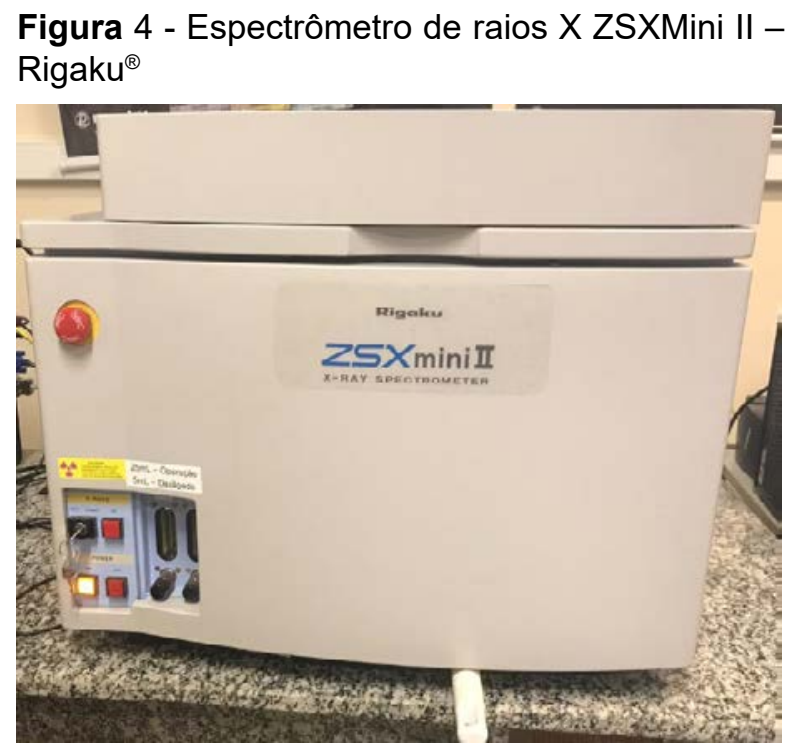

Fonte: Freitas Filho, 2019.

\subsubsection{DRX}

A difratometria das amostras policristalinas foi realizada na Panalytical, modelo X'Pert Pro MPD®, ilustrada na Figura 5, com tensão e corrente de operação de 40 kV x 40 mA, radiação CoKa, e o feixe incidente combinado com um monocromador híbrido, composto de um espelho parabólico e dois cristais de germânico (220), onde se objetiva encontrar um difratograma correspondente da amostra e realizar a identificação das fases dos compostos químicos presentes (FREITAS FILHO, 2019.). A técnica DRX utiliza a propriedade da difração aplicada aos raios $\mathrm{X}$, com isso possibilita a obtenção de importantes informações referentes à rede cristalina, tais como a identificação e a quantificação das fases presentes em um sistema policristalino multifásico, análise do tamanho do cristalito e a deformação da rede cristalina. Além disso, é analisada a ocupação de sítios cristalográficos por dopantes adicionados, a obtenção das distâncias interatômicas e outros fatores referentes à estrutura cristalina (CULLITY, 1978). 
Figura 5 - X'Pert Pro MPD ${ }^{\circledR}$

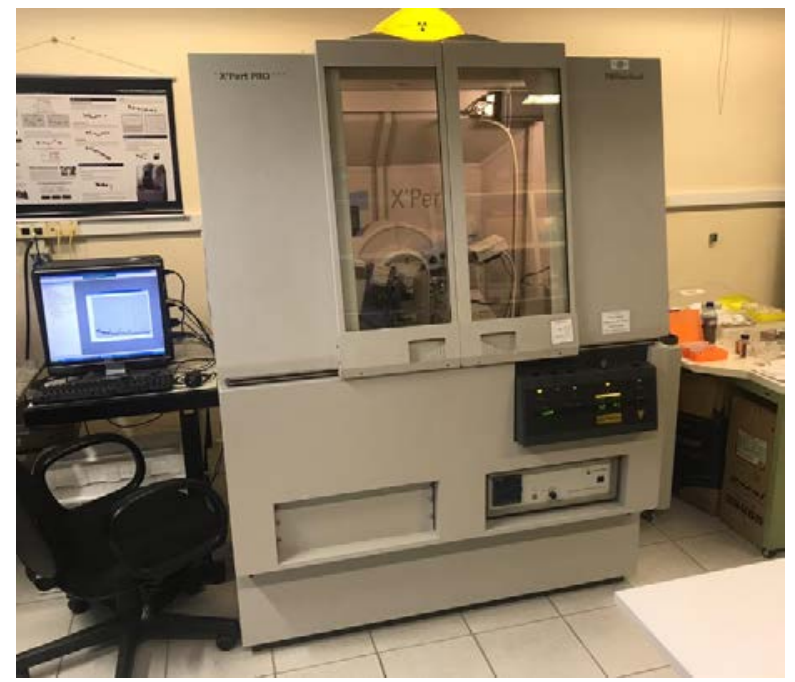

Fonte: Freitas Filho, 2019.

Em relação ao ângulo de entrada medido (2Ө), foi definido um intervalo de $10^{\circ}$ a $100^{\circ}$, com uma variação angular de $0,013^{\circ}(2 \Theta)$, um passo que permite uma maior precisão e um intervalo abrangente, baseados no trabalho de Costa et al. (2016) e na previsão das possíveis fases a serem medidas.

\subsubsection{MEV e EDS}

A microscopia e a espectrometria, realizadas na Central Analítica, foram realizadas com o microscópio eletrônico de varredura da FEI Company ${ }^{\circledR}$, modelo Quanta 450 FEG, observado na Figura 6, obtendo-se uma análise qualitativa (identificação da distribuição das partículas ao longo da amostra) e quantitativa (tamanho de cada partícula), objetivando a determinação do tamanho das partículas das amostras coletadas.

Figura 6 - Microscópio eletrônico de varredura da FEI Company®, modelo Quanta 450 FEG

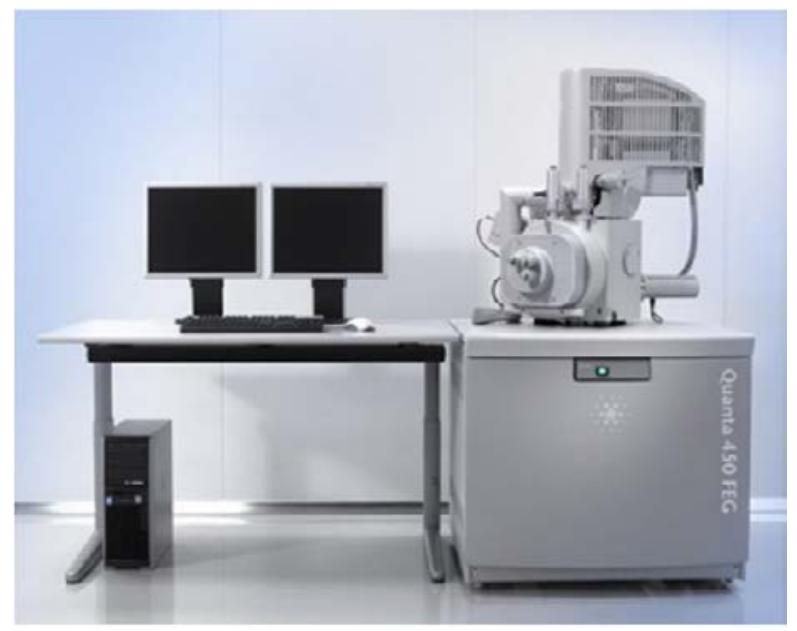

Fonte: Manual MEV Quanta 450 FEG, 2015.

Para a realização do ensaio, foi extraída uma pequena quantidade da amostra coletada, que foi colocada sob uma fita de carbono e uma superfície de isopor (Figura 7). Tal procedimento é utilizado quando a amostra não é eletricamente condutora, pois o acúmulo de cargas elétricas em sua superfície dificulta, ou mesmo impossibilita, a obtenção de imagens de elétrons secundários. Para contornar esse efeito, existe a possibilidade 
de fazer um recobrimento metálico (geralmente ouro) ou de carbono para tornar a superfície condutora (Manual MEV QUANTA 450 FEG, 2015). Nesse microscópio, foram realizadas observações e o detalhamento do tamanho das partículas, por meio do MEV, e da composição das amostras, por meio do EDS.

Figura 7 - Preparação das amostras para o MEV e o EDS

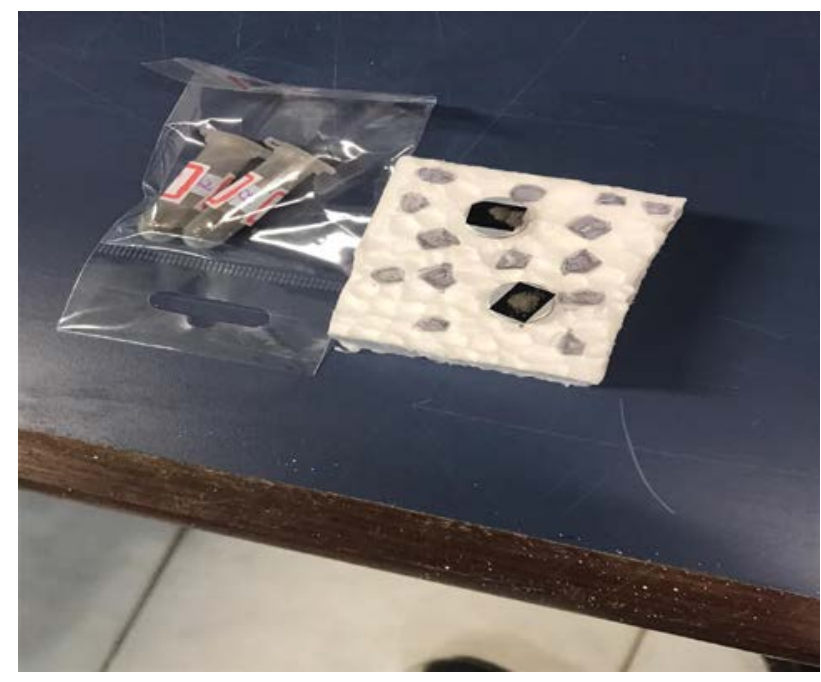

Fonte: Elaborada pelos autores, 2020.

O princípio de um microscópio eletrônico de varredura consiste em utilizar um feixe de elétrons de pequeno diâmetro para explorar a superfície da amostra, ponto a ponto, por linhas sucessivas, e transmitir o sinal do detector a uma tela catódica cuja varredura está perfeitamente sincronizada com aquela do feixe incidente. Por um sistema de bobinas de deflexão, o feixe pode ser guiado de modo a varrer a superfície da amostra segundo uma malha retangular. O sinal de imagem resulta da interação do feixe incidente com a superfície da amostra. O sinal recolhido pelo detector é utilizado para modular o brilho do monitor, permitindo a observação. A maioria dos instrumentos usa como fonte de elétrons um filamento de tungstênio $(W)$ aquecido, operando numa faixa de tensões de aceleração de 1 a $50 \mathrm{kV}$. O feixe é acelerado pela alta tensão criada entre o filamento e o ânodo. Ele é, em seguida, focalizado sobre a amostra por uma série de três lentes eletromagnéticas com um spot menor que $4 \mathrm{~nm}$. O feixe interagindo com a amostra produz elétrons e fótons que podem ser coletadas por detectores adequados e convertidas em um sinal de vídeo (DEDAVID; GOMES; MACHADO, 2007).

\section{Resultados e discussão}

\subsection{FRX}

A análise elementar foi realizada utilizando o princípio do FRX, como visto na Tabela 2, em que se observa uma maior presença dos elementos químicos alumínio (Al), silício ( $\mathrm{Si}$ ), cálcio (Ca), ferro (Fe), potássio $(\mathrm{K})$ e cloro $(\mathrm{Cl})$ nas amostras coletadas. Alguns elementos químicos, como o carbono, não aparecem nos resultados, pois o equipamento utilizado abrange a um intervalo dos elementos químicos do flúor ao urânio (FREITAS FILHO, 2019).

Segundo Morawska e Zang (2001), compostos como Al e Fe são comumente associados aos transportes rodoviários, especificamente do desgaste do motor. Além disso, $\mathrm{Al}, \mathrm{Si}, \mathrm{K}, \mathrm{Ca}$, Titânio (Ti) e Fe podem ser originários da poeira das rodovias, comuns no processo de queima de combustíveis fósseis. Além disso, $\mathrm{Si}, \mathrm{Ti}, \mathrm{Ca}, \mathrm{Al}$, $\mathrm{Zn}$ e $\mathrm{Mn}$ podem ter origem em partículas em suspensão advindas do solo. A presença de $\mathrm{Cl}$ é possivelmente oriunda da salinidade, já que as plantas FV estão localizadas em uma região litorânea, indicando a possível 
presença de sódio $(\mathrm{Na})$, elemento de difícil identificação nesse equipamento devido à baixa energia emitida, podendo, portanto, haver a presença de cloreto de sódio (NaCl) (FREITAS FILHO, 2019).

Tabela 2 - Dados do FRX.

\begin{tabular}{c|c|c}
\hline Elemento químico & $\begin{array}{c}\text { Porcentagem em massa planta FV 1 } \\
(\mathbf{\%})\end{array}$ & $\begin{array}{c}\text { Porcentagem em massa planta FV } \\
\mathbf{2} \text { (\%) }\end{array}$ \\
\hline $\mathrm{Al}$ & 8,8692 & 10,3060 \\
\hline $\mathrm{Si}$ & 36,6740 & 40,2330 \\
\hline $\mathrm{P}$ & 0,4322 & 0,5036 \\
\hline $\mathrm{S}$ & 0,9433 & 0,6923 \\
\hline $\mathrm{Cl}$ & 4,1792 & 2,8078 \\
\hline $\mathrm{K}$ & 6,7172 & 6,4511 \\
\hline $\mathrm{Ca}$ & 18,2730 & 17,1800 \\
\hline $\mathrm{Ti}$ & 2,3395 & 2,7184 \\
\hline $\mathrm{Mn}$ & 0,6313 & 0,5592 \\
\hline $\mathrm{Fe}$ & 19,5480 & 17,2820 \\
\hline $\mathrm{Cu}$ & 0,1250 & 0,1741 \\
\hline $\mathrm{Zn}$ & 0,8499 & 0,6205 \\
\hline $\mathrm{Sr}$ & 0,1228 & 0,1349 \\
\hline $\mathrm{Zr}$ & 0,2955 & 0,3366 \\
\hline
\end{tabular}

Fonte: Freitas Filho, 2019.

\subsection{DRX}

A análise dos resultados do $D R X$, Figura 8, foi feita utilizando o software X-Pert Highscore Plus, Panalytical B.V. Almelo, Holanda, (C) 2001 Koninklijke Philips Electronics N.V (All rights reserved), que possibilita a identificação das fases correspondentes aos compostos químicos. A análise indicou os seguintes componentes com alta probabilidade de ocorrência: $\mathrm{SiO}_{2}$ (códigos de referência: 01-086-1629 e 01-082-1410), que pode ter como fonte a areia; $\mathrm{Fe}_{2} \mathrm{O}_{3}$ (código de referência: 00-052-1449), que pode ter como origem de elementos ferrosos; $\mathrm{NaCl}$ (código de referência: 96-100-0042), possivelmente oriundo da salinidade oriunda da região, já que Fortaleza é uma cidade litorânea; $\mathrm{KAl}_{2}\left(\mathrm{AlSi}_{3} \mathrm{O}_{10}\right)(\mathrm{OH})_{2}$ (código de referência: 00-026-0911), mineral do grupo dos silicatos, que pode ter como origem rochas sedimentares; $\mathrm{C}_{6} \mathrm{H}_{8} \mathrm{O}_{6}$, (código de referência: 00-0461983), um composto de origem orgânica; $\mathrm{CaCO}_{3}$ (código de referência:.00-005-0586), comumente oriundo de rochas. Há de se destacar, novamente, a possível presença de mais compostos orgânicos ou inorgânicos com a presença de carbono, porém a análise não forneceu resultados concretos que indicassem a presença de compostos específicos (SCHWELA; MORAWSKA; KOTZIAS, 2002; FREITAS FILHO, 2019). 
Figura 8 - Difratograma da amostra das plantas 1 e 2 respectivamente
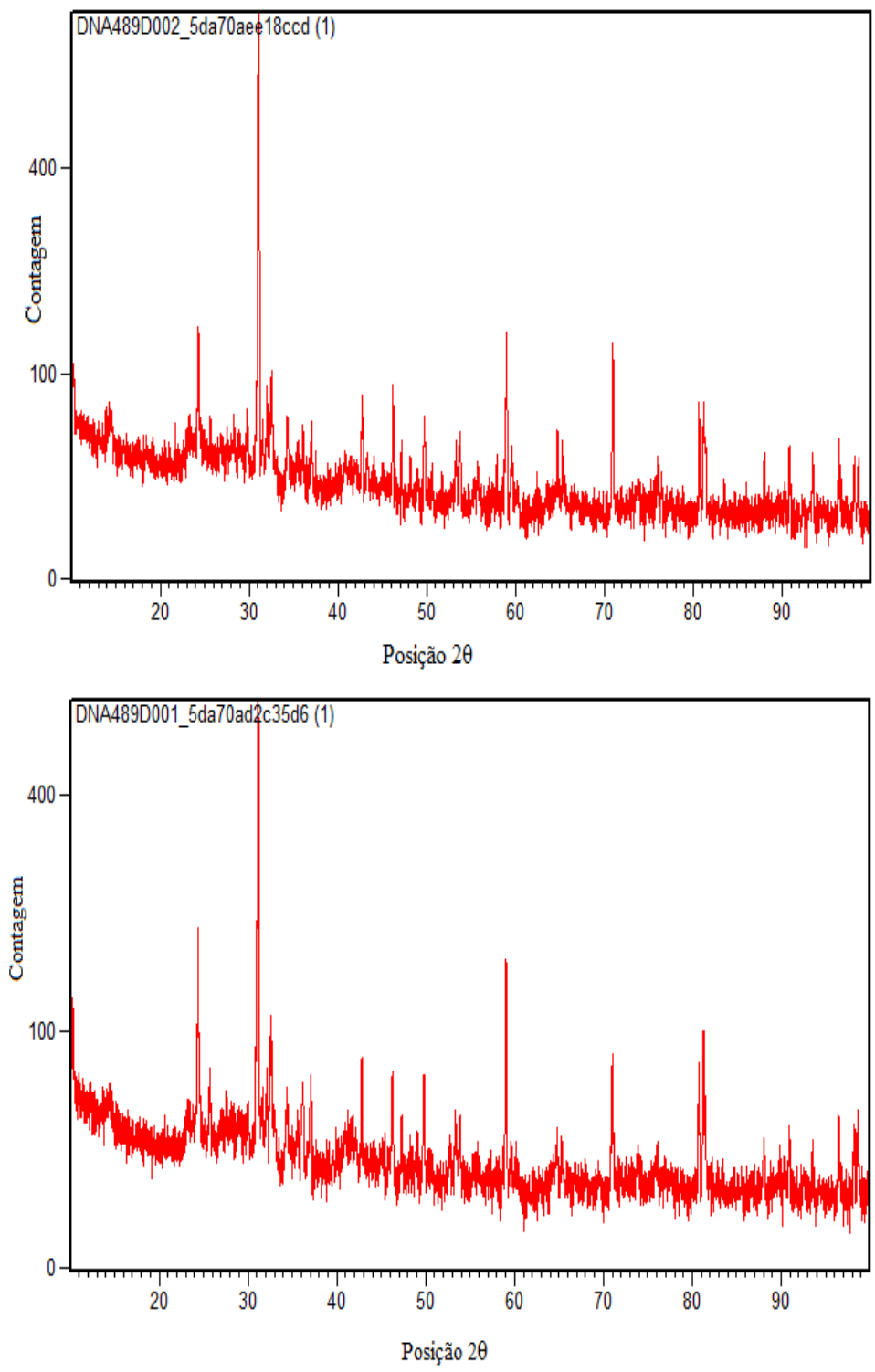

Fonte: Adaptado do software X-Pert Highscore Plus (2019).

Os resultados obtidos da análise da planta 1 se assemelham bastante com os da planta 2, diferindo apenas na intensidade dos picos. Tal fato era esperado, pois as plantas localizam-se no mesmo telhado, possuindo mesma inclinação $\left(10^{\circ}\right)$. Além disso, os picos próximos aos $10^{\circ}$ apresentam um comportamento semelhante ao de compostos argilosos.

\subsection{MEV}

Os resultados do MEV na planta FV 1 confirmam uma alta variabilidade no tamanho das partículas, desde $14 \mu \mathrm{m}$ até $112,1 \mu \mathrm{m}$, havendo uma predominância de partículas de $10 \mu \mathrm{m}$ até $40 \mu \mathrm{m}$. Observa-se, na Figura 9, locais mais claros nas amostras, o que indica a presença de partículas eletricamente carregadas. Além disso, é possível observar que há locais sem a presença de partículas, onde se pode atestar a presença da fita de carbono (C), como observado na Figura 7. 
Figura 9 - MEV da amostra da planta FV 1

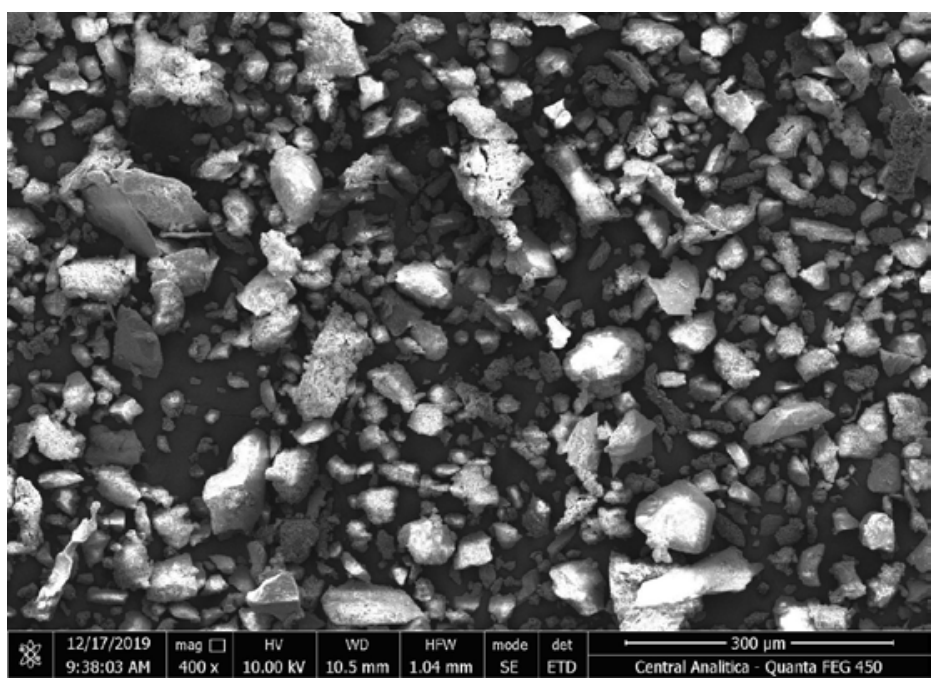

Fonte: Adaptado do Microscópio Eletrônico de Varredura $\mathrm{FEI}^{\circledR}$ Quanta 450 FEG (2020)

Os resultados do MEV na planta FV 2 são semelhantes aos da planta FV 1, com variabilidade do tamanho das particular, porém com uma maior presença das partículas maiores, desde 11,31 $\mu \mathrm{m}$ até $208,3 \mu \mathrm{m}$, como atesta a Figura 10.

Figura 10 - MEV da amostra da planta FV 2

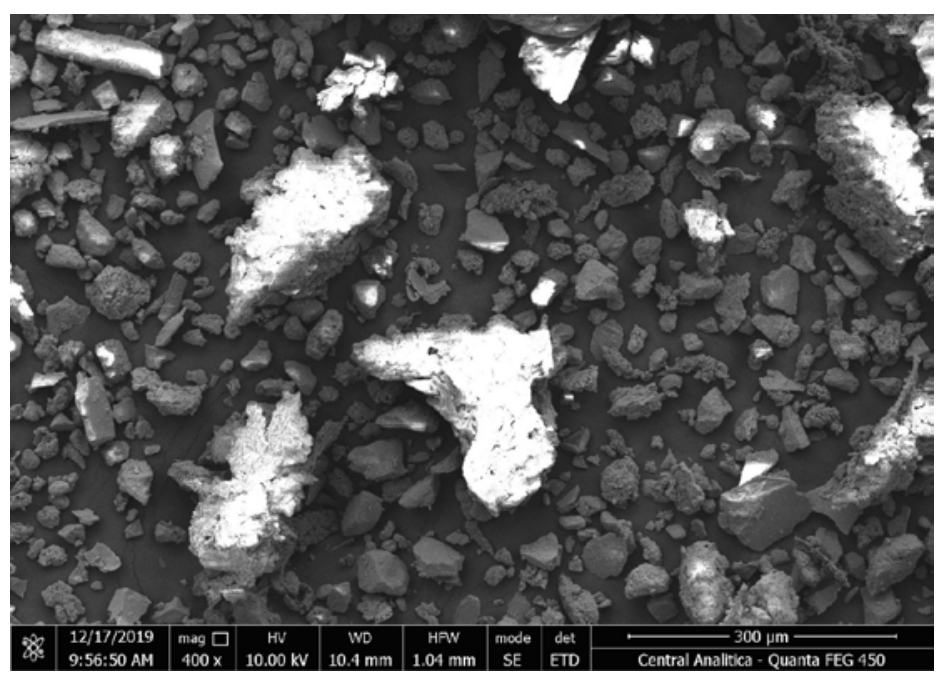

Fonte: Adaptado do microscópio eletrônico de varredura da FEI Copany®, modelo Quanta 450 FEG (2020)

Observa-se que, na amostra da Figura 10, o tamanho das partículas é mais uniforme, havendo também mais espaços vazios, o que pode implicar numa maior presença de carbono decorrente da fita do que da planta FV 1.

\subsection{EDS}

Em relação ao EDS da planta FV 1, cujo resultado é observado na Figura 11, nota-se a presença de ouro, porém, no cálculo da porcentagem de participação de cada elemento químico presente, ele não foi incluído, pois foi adicionado ouro no processo de metalização. Já a presença de $C$ pode ser menor do que indicam os resultados, devido ao carbono contido na fita e a presença de espaços vazios (onde há presença da fita) 
na amostra. Nota-se que os resultados de presença de elementos químicos são semelhantes aos do FRX, havendo a adição dos elementos químicos oxigênio $(\mathrm{O}), \mathrm{C}$ e Na, que não foram detectados nos ensaios de FRX, devido ao pequeno raio atômico ou por não abranger o intervalo que o equipamento atua. Além disso, a análise atesta a presença de ouro $(\mathrm{Au})$, que foi excluído do cálculo percentual, pois sua presença decorre da etapa de metalização da amostra, não sendo, portanto, resíduo da amostra original.

Figura 11 - EDS da amostra da planta 1

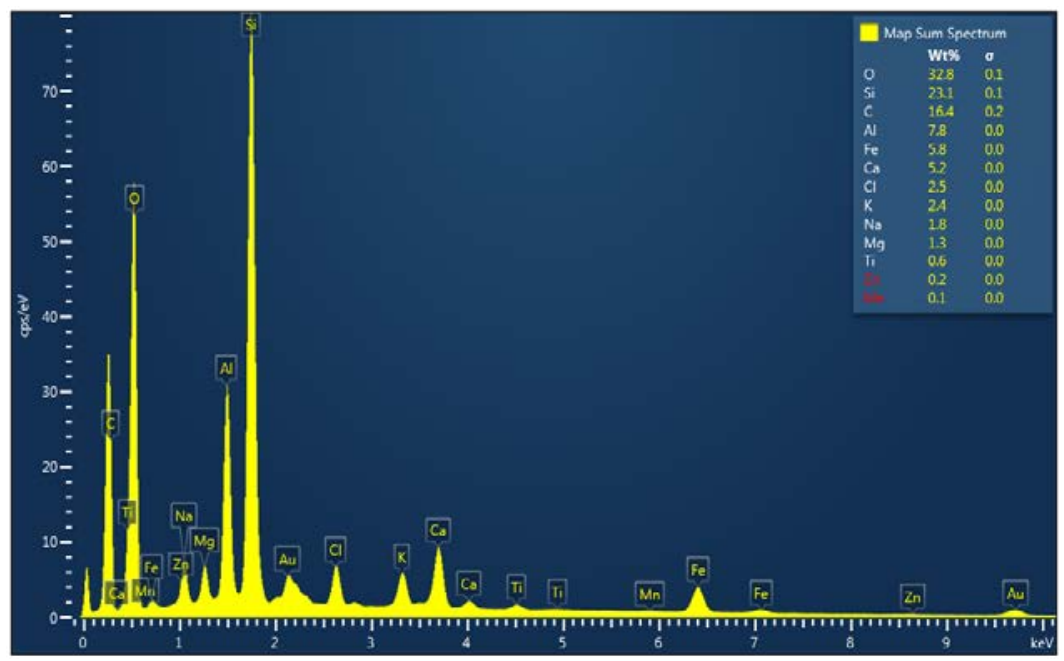

Fonte: Elaboração dos próprios autores a partir do EDS, 2020.

Já a Figura 12 mostra a composição da amostra da planta FV 2, em que se nota a presença de $\mathrm{Si}, \mathrm{Al}$, $\mathrm{Fe}, \mathrm{Ca}, \mathrm{K}, \mathrm{Cl}, \mathrm{Zn}, \mathrm{Mg}$, Ti e Mn, semelhante aos resultados do FRX, o que atesta a validade do ensaio.

Figura 12- EDS da amostra da planta 2

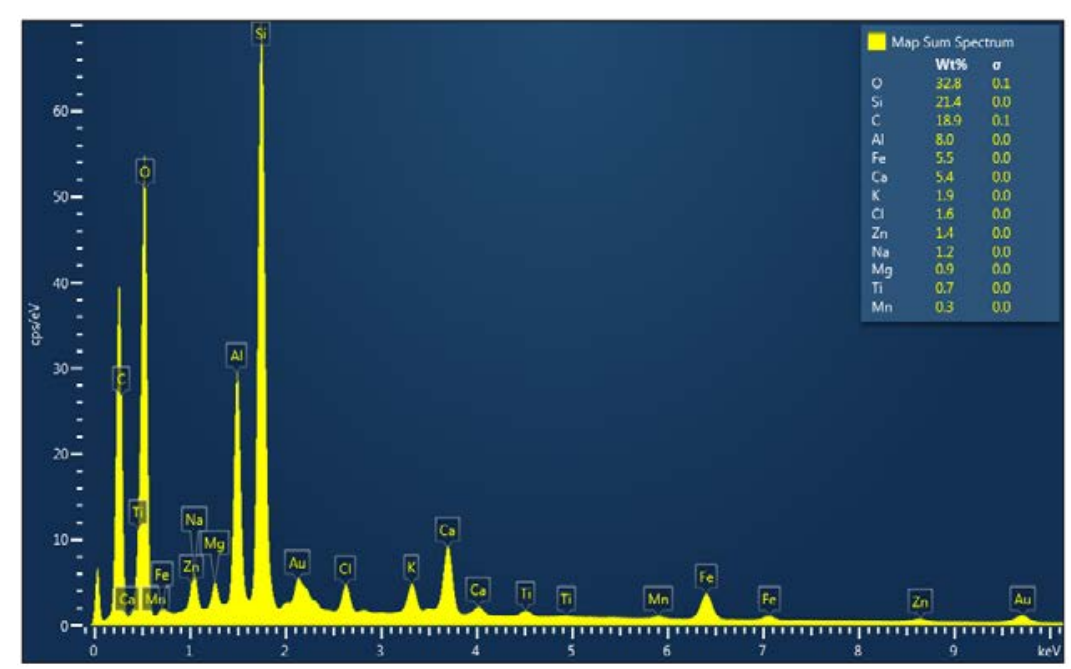

Fonte: Elaboração dos próprios autores a partir do EDS, 2020.

\section{Conclusão}

No presente artigo foi analisada a composição da sujidade presente em duas plantas FV conectadas à rede elétrica no LEA-UFC, campus do Pici, em Fortaleza. Foram analisados diversos artigos na área no estado da arte, porém foi verificada a falta de estudos sobre plantas FV localizadas na região Nordeste do 
Brasil, onde há grande incidência de irradiação solar e muitos sistemas FV têm sido instalados. Os ensaios de caracterização da sujidade realizados ajudam a compreender a composição da sujidade presente nos módulos FV e o tamanho das partículas. Como característica climática de Fortaleza, a quantidade de precipitação no primeiro semestre do ano ajuda no processo de limpeza natural, não havendo grande acúmulo de sujidade.

As plantas FV estudadas estão localizadas no mesmo telhado e, por isso, contêm composição de sujidade bastante similar, como atestam os resultados com o uso do FRX e do EDS. Além disso, foi possível constatar que a composição da sujidade condiz com a literatura, pois as plantas estão localizadas ao lado de uma avenida com intenso tráfego de veículos. Utilizando o FRX, foram encontrados elementos químicos, como $\mathrm{Si}, \mathrm{Al}, \mathrm{Fe}, \mathrm{Ca}$ e Ti, que podem ser decorrentes da sujidade oriunda da avenida. Elementos como $\mathrm{O}, \mathrm{C}$ e $\mathrm{Na}$ foram identificados na EDS e não foram identificados na FRX, devido à diferença dos princípios e do intervalo que os equipamentos utilizados abrangem.

Os resultados obtidos pelo DRX fornecem dados que podem ser estudados por meio da análise de conjuntos de picos, utilizando o software $X^{\prime} P$ ert HighScore ${ }^{\circledR}$. É importante ressaltar que as análises são qualitativas. Desse modo, para a confirmação das fases cristalinas e sua concentração, é necessária a realização de um refinamento estrutural (método de Rietveld). Portanto, as prováveis fases cristalinas encontradas nas plantas FV analisadas foram: $\mathrm{SiO}_{2}, \mathrm{Fe}_{2} \mathrm{O}_{3}, \mathrm{NaCl}, \mathrm{KAl}_{2}\left(\mathrm{AlSi}_{3} \mathrm{O}_{10}\right)(\mathrm{OH})_{2}, \mathrm{CaCO}_{3}$ e $\mathrm{C}_{6} \mathrm{H}_{8} \mathrm{O}_{6}$.

Os resultados do MEV confirmaram que o tamanho das partículas encontradas é bem semelhante e está na escala dos micrometros, o que, dependendo das características de adesão das partículas à superfície dos módulos FV, pode dificultar o processo de limpeza.

\section{Referências}

ARAÚJO, Danielly Norberto; CARVALHO, Paulo Cesar Marques; DUPONT, Ivonne Montero. Efeitos da acumulação de sujeira sobre o desempenho de módulos fotovoltaicos. Revista Tecnologia, Fortaleza, v. 40, n. 2, 2019. DOI: http://dx.doi.org/10.5020/23180730.2019.9414.

CALLISTER, William D.; RETHWISCH, David G.Ciência e engenharia de materiais:uma introdução. 8. ed. Rio de Janeiro, RJ: Livros Técnicos e Científicos, 2012.

CONCEIÇÃO, R.; et al. Saharan dust transport to Europe and its impact on photovoltaic performance: A case study of in Portugal. Solar Energy, [S. I.], v. 160, 2018, p. 94-102, 2018. Disponível em: https://doi. org/10.1016/j.solener.2017.11.059.

COSTA, S. C.; DINIZ, A. S. A.; KAZMERSKI, L. L. Solar energy dust and r\&d progress: literature review update for 2016. Renewable and Sustainable Energy Reviews, [S. I.], v. 82, p. 2504-2536, 2018.

COSTA, S. C. S.; et al. Caracterização físico-química da sujidade depositada sobre módulos fotovoltaicos instalados em zonas climáticas de Minas Gerais. In: CONGRESSO BRASILEIRO DE ENERGIA SOLAR, 6. ., 2016, Belo Horizonte. Anais [...]. Belo Horizonte: [s. n.], 2016. p. 1-9.

CULLITY, D. B. Elements of X-Ray Diffraction. Addison-Wesley Publishing company inc, 1978.

DEDAVID, B. A.; GOMES, C. I.; MACHADO, G. Microscopia eletrônica de varredura aplicações e preparação de amostras. Porto Alegre: EDIPURGS, 2007.

EMPRESA DE PESQUISA ENERGÉTICA (EPE). Balanço energético nacional: 2019. Disponível em: http://www.epe.gov.br/pt/publicacoes-dados-abertos/publicacoes/balanco-energetico-nacional-ben. Acesso em: 20 mar. 2020.

9FERRADA, P.; et al. Physicochemical characterization of from photovoltaic facilities in arid locations in the Atacama Desert. Solar Energy, [S. I.], v. 187, p. 47-56, mayo, 2019. Disponível em: https://doi. org/10.1016/j.solener.2019.05.034. 
FREIRE, R. S.; LIMA, P. H. M.; MIGUEL, E. C. Manual de operação MEV QUANTA 450 FEG. Fortaleza: UFC, 2015.

FREITAS FILHO, M. F. Análise da composição da sujidade em plantas fotovoltaicas instaladas na zona urbana de Fortaleza. 2019. Trabalho de Conclusão de Curso (Graduação em Engenharia de Energias Renováveis) - Universidade Federal do Ceará, Fortaleza, 2019.

GHOLAMI, A.; et al. Experimental investigation of dust deposition effects on photo-voltaic output performance. Solar Energy, [S. I.], v. 159, 2017, p. 346-352, nov. 2018. Disponível em: https://doi. org/10.1016/j.solener.2017.11.010.

HACHICHA, A. A.; AL-SAWAFTA, I.; SAID, Z. Impact of dust on the performance of solar photovoltaic (PV) systems under United Arab Emirates weather conditions. Renewable Energy, [S. I.], v. 141, p. 287-297, 2019. Disponível em: https://doi.org/10.1016/j.renene.2019.04.004.

IEA. International Energy Agency, Snapshot of global markets: 2019. Disponível em: http://www.iea-pvps. org/fileadmin/dam/public/report/statistics/IEA-PVPS_T1_35_Snapshot2019-Report.pdf. Acesso em: 20 mar. 2020.

JAVED, W.; WUBULIKASIMU, Y.; FIGGIS, B.; GUO, B. Characterization of dust accumulated on photovoltaic panels in doha, qatar. Solar Energy, [S. I.], v. 142, p. 123-135, 2017.

KAZEM, H. A.; CHAICHAN, M. T. Experimental analysis of the effect of dust's physical properties on photovoltaic modules in northern oman. Solar Energy, [S. I.], v. 139, p. 68-80, 2016.

LAWRENCE, C. R.; NEFF, J. C. The contemporary physical and chemical flux of aeolian dust: a synthesis of direct measurements of dust deposition. Chemical Geology, [S. I.], v. 267, n. 1-2, p. 46-63, 2009.

MENOUFI, K. et al. Dust accumulation on photovoltaic panels: A case study at the East Bank of the Nile (Beni-Suef, Egypt). Energy Procedia, [S. I.], v. 128, p. 24-31, 2017. Disponível em: https://doi.org/10.1016/j. egypro.2017.09.010.

MICHELE, L., DECEGLIE, M. G., e MULLER, M. (2019). Predicting photovoltaic soiling losses using environmental parameters: An update. Progress in Photovoltaics: Research and Applications, vol. 27, n. 3, pp. 210-219.

MORAWSKA, L.; ZANG J. Combustion sources of particles: health relevance and source signatures. Chemosphere, [S. I.], v. 49, n. 9, p. 1045-1057, dec. 2001.

ROMANHOLO, P. V. V.; et al. Sujidade depositada sobre módulos fotovoltaicos instalados em Goiânia: morfologia e composição química. Energia solar e eólica 2. In: CONGRESSO BRASILEIRO DE ENERGIA SOLAR, 7., 2018, Gramado. Anais, [...]. 2018, Gramado: [s.n.], 2018. p. 1-8.

SAIDAN, M.; ALBAALI, A. G.; ALASIS, E.; KALDELLIS, J. K. Experimental study on the effect of dust deposition on solar photovoltaic panels in desert environment. Renewable Energy, [S. I.], v. 92, p. 499505, 2016.

SARVER, T.; AI QARANGHULI, A.; KAZMERKI, L. A comprehensive review of the impact of dust on the use of solar energy: History, investigations, results, literature, and mitigation approaches. Renewable and Sustainable Energy Reviews. 2013; 22 (60): 698733.

SCHWELA, D. H.; MORAWSKA, L.; KOTZIAS, D. (ed.). Guidelines for concentration and exposureresponse measurement of fine and ultra fine particulate matter for use in epidemiological studies. Geneva: World Health Organization, 2002.

TANESAB, J.; et al. The effect of dust with different morphologies on the performance degradation of photovoltaic modules. Sustainable Energy Technologies and Assessments, v. 31, p. 347-354, 2019. 


\section{Agradecimentos}

Ao CNPq, pela bolsa de produtividade em pesquisa 2 para o terceiro autor.

Ao Laboratório de Energias Alternativas (LEA) da UFC

Ao Laboratório de Raios-X (LRX) da UFC.

À Central Analítica da UFC.

\section{Sobre os autores}

\section{Marcelo Ferreira Freitas Filho}

Graduando em Engenharia de Energias Renováveis pela Universidade Federal do Ceará (UFC).

\section{Danielly Norberto Araujo}

Mestra (2020) em Engenharia elétrica pela Universidade Federal do Ceará (UFC). Graduada (2017) em Engenharia Elétrica pela Universidade Federal de Campina Grande (UFCG). Foi pesquisadora no Laboratório de Energias Alternativas da UFC (2018-2020). Atualmente, é pesquisadora no Instituto de Tecnologia Edson Mororó Moura. Seus interesses são: geração distribuída, energia solar e sistemas de armazenamento de energia.

\section{Paulo Cesar Marques de Carvalho}

Doutor em Engenharia Elétrica pela Universidade de Paderborn, Alemanha (1997). Mestre em Engenharia Elétrica pela Universidade Federal da Paraíba (1992). Graduado em Engenharia Elétrica pela Universidade Federal do Ceará (1989). Atualmente, é professor titular do Departamento de Engenharia Elétrica da Universidade Federal do Ceará. Tem atividades de ensino, pesquisa e extensão nos temas: geração fotovoltaica, geração eólica e biodigestores. Coordena o Laboratório de Energias Alternativas da UFC. Bolsista de produtividade em pesquisa do CNPq.

\section{José Marcos Sasaki}

Doutor em Física pelo Instituto de Física da Unicamp (1993). Mestre em Física (1988). Graduado (1983) (Licenciatura plena) em Física pela Universidade Estadual de Maringá (UEM). Desde abril de 2016 é professor titular do Departamento de Física da Universidade Federal do Ceará. Tem experiência na área de Física, atuando principalmente nos seguintes temas de pesquisa: teoria dinâmica da difração de raios-x, nanopartículas e método Rietveld. É coordenador do Laboratório Multiusuário em Nanotecnologia/CNPq. Atualmente, trabalha na caracterização de cristais por difração de raios-X usando radiação síncrotron e na síntese de nanopartículas aplicadas em sensores ópticos e células fotovoltaicas. Publicou 121 artigos com 1840 citações (Web of Science) com Fator $\mathrm{H}=24$.

Recebida em: 09.04.2020

Aceita em: 09.06.2020 